\title{
THE NOSE IN RELATION TO THE CEREBROSPINAL FLUID AND LYMPH STREAM
}

\author{
BY \\ J. M. YOFFEY, D.Sc., M.D., F.R.C.S. \\ (From the Department of Anatomy, University of Bristol)
}

Introduction

In health the nose is the first part of the respiratory tract to receive the impact of air from outside, and the structure of the nasal mucous membrane is well adapted to respond to some of the changes in the composition and properties of this air. The response consists in part of saturating the inspired air with water vapour, and adjusting its temperature to that of the body; it also is instrumental in holding up particulate matter, and preventing a good deal of it from reaching the remainder of the respiratory pathway and lungs. If the air is cold it is warmed before it reaches the lungs, and for this reason the nasal mucosa is endowed with a high degree of vascularity, being rich not only in capillaries, but also in an abundance of arteriovenous anastomoses, in virtue of which it is capable both of rapid engorgement and of equally rapid shrinkage. If the inspired air is dry it is moistened as it is drawn through the nose, but the mechanism of this moistening is somewhat obscure. Though the nasal mucosa is rich in mucous glands, the moistening of the air requires not a mucous but a watery secretion or possibly transudation. The secretion of the healthy mucous membrane does in fact contain very little mucus, and it requires an abnormal irritant to evoke the full mucous secretion of which the nasal epithelium is capable.

Particulate matter is to some extent held back by the nose, partly by the hairs in the proximity of the external nares, partly by the adhesive properties of the moist surface of the mucosa, the adherent particles being subsequently removed by increased secretion and ciliary action. If the particles are inanimate, for example, soot particles in a fog, this mechanism is fairly adequate. But if the particles are animate, such as viruses or bacteria, then if they are pathogenic they can invade the mucosa and multiply in it. Once they have invaded the mucosa,

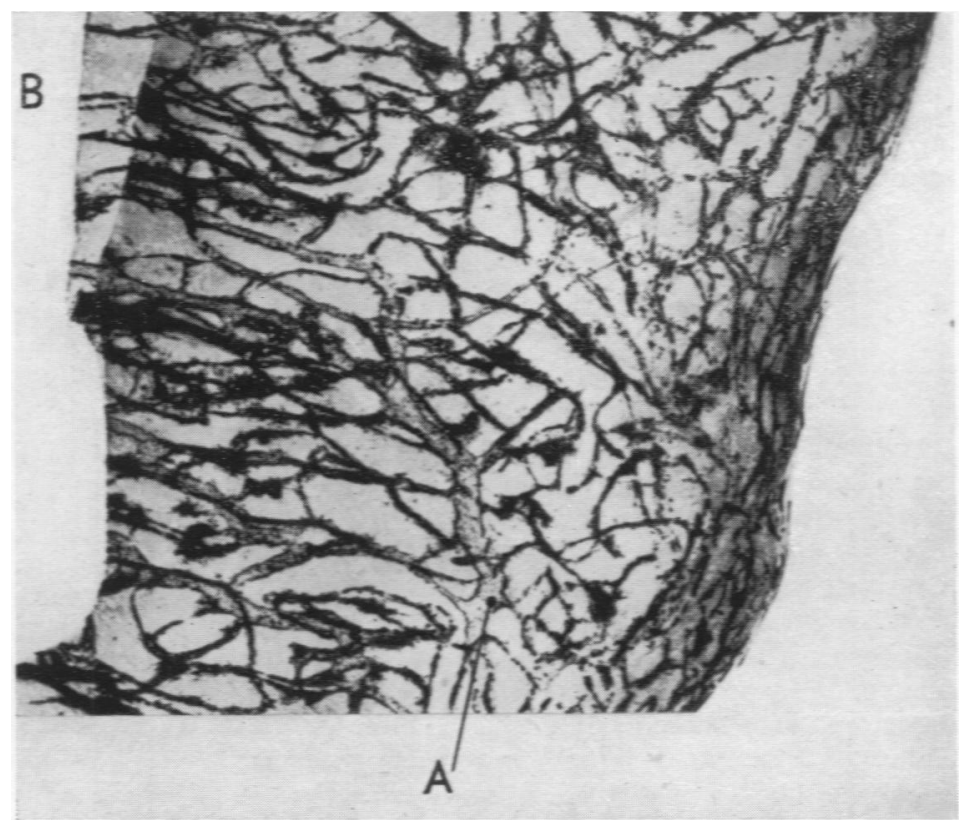

FIG. 1 ( 50$)$. - One of the turbinate bones from the same specimen as fig. 2. The smaller vessels are completely filled with ink, whereas in the larger vessels the lumen is almost empty, and the ink has adhered to the walls. The line marked $A$ ends in one of these larger vessels. The turbinate bone has been cleared by the Spalteholtz technique, and then photographed by transmitted light. The plexus is present on both aspects of the bone, but in the top left hand corner, where the turbinate has been cut obliquely, the plexus on one side only is seen (B). 


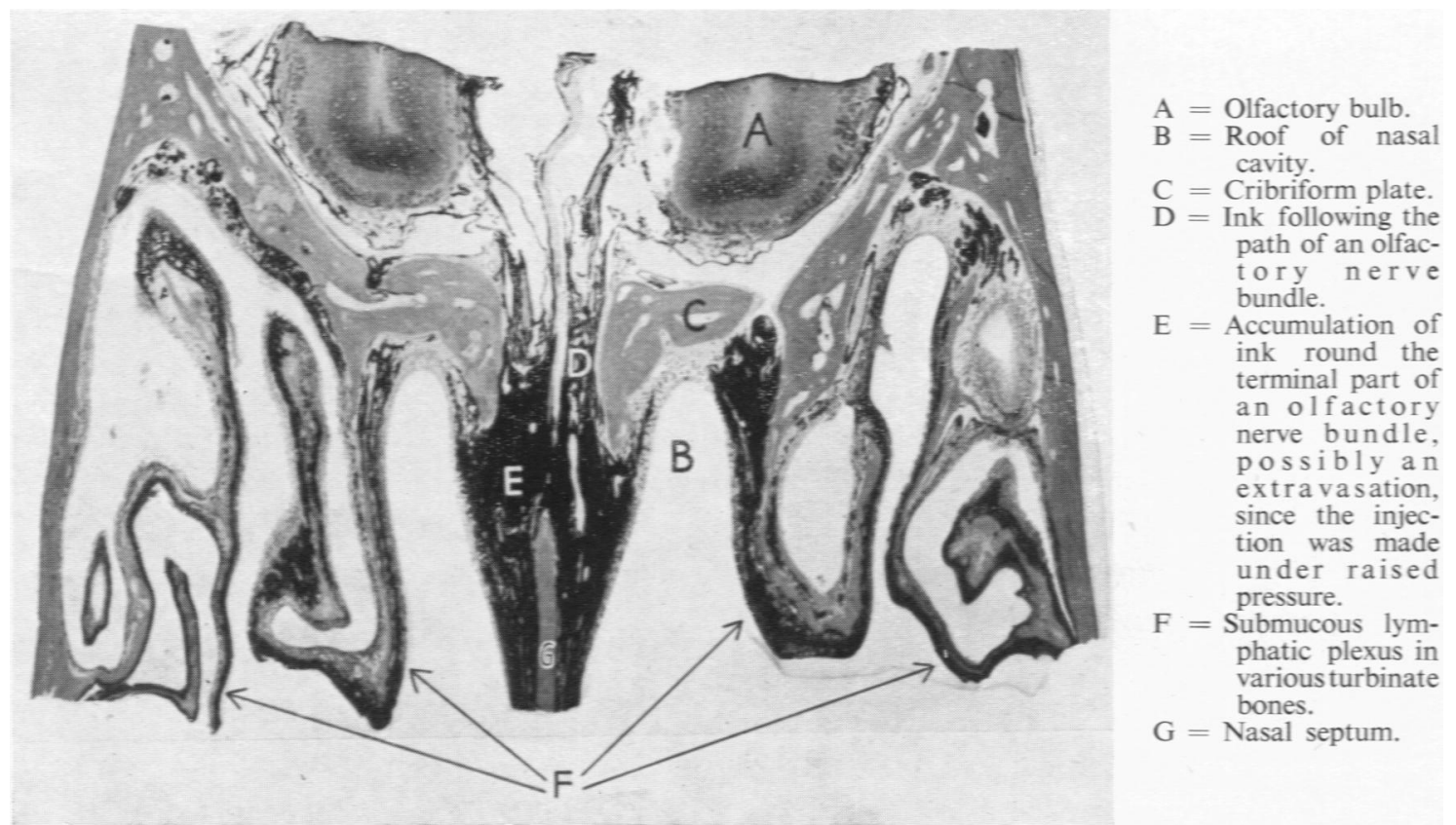

FIG. $2(10)$. - Coronal section through the roof of the nose in a cat which had received under raised pressure an injection of indian ink into the cranial subarachnoid space. This shows the pathway taken by ink from the interior of the cranium to the submucous lymphatic plexus of the nose and the cervical pathway.

deeply and enter the submucous tissues. From the submucosa, viruses and bacteria can spread not only by direct extension, but also more rapidly and further afield through lymphatic and blood vessels, as well as along the paths provided by bundles of nerve fibres.

The submucous lymphatic plexus of the nose is unusually well developed. When a richly vascular area is subjected to rapid changes in its vascularity, and when, furthermore, its blood vessels are frequently exposed to such varying degrees of irritation, with consequent increase in the permeability of their endothelium, it is not surprising that there should on occasion be a rapid outpouring of fluid in its tissue spaces, giving rise to swelling and oedema which are not to be confused with vascular engorgement, though the two frequently co-exist. In the narrower respiratory passage of infancy, a relatively small amount of oedema will suffice to cause appreciable obstruction to the flow of air. Since it is important that the respiratory passages should be kept as clear and unobstructed as possible it is to be expected that a rich lymphatic plexus should be present in the submucosa, to facilitate rapid clearance of this oedema fluid. Fig. 1 illustrates the submucous plexus from one of the turbinate bones of a cat's nose, after injection with indian ink. From the point of view of illustrating the nature and density of the plexus, it is immaterial that this particular injection was made under pressure from the cranial subarachnoid space (fig. 2).

\section{The Lymphatic Pathway}

The rich submucous lymphatic plexus is drained by a well-defined lymphatic pathway, which can be demonstrated with great ease and in a most convincing manner by the instillation into the nose of a solution of vital dye (Yoffey and Drinker, 1938). Trypan blue is perfectly satisfactory, though Evans blue (T-1824), an isomer of trypan blue, is even better because of its more intense colour. The vital dye traverses the living mucous membrane and then enters the submucous lymphatics, whence it is drained by a number of collecting trunks descending from the posterior and lateral walls of the nasopharynx. These converge as they descend, and in most mammals drain into a single large lymph gland, close to the upper end of the internal jugular vein. From the lower end of this gland a single efferent vessel emerges, the deep cervical duct, or jugular trunk. This descends in close proximity to the internal jugular vein, and ends by opening on the right side into the right lymph duct, on the left side into the thoracic duct. The arrangement in the monkey (fig. 3) and man is essentially similar, except that the course of the lymph is interrupted by a chain of six or more small glands instead of a single large one. 


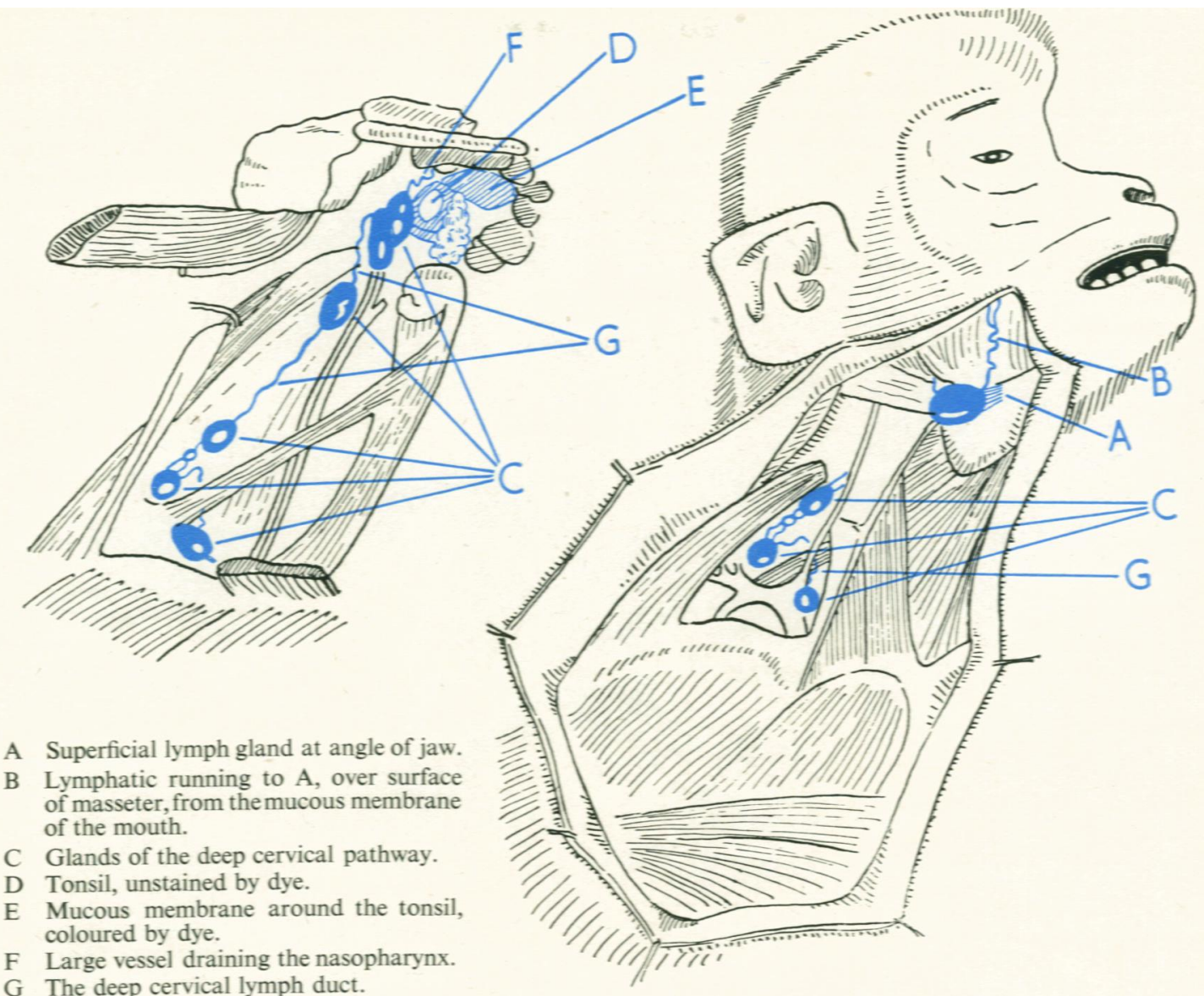

FIG. 3.

The cervical lymphatic pathway in the monkey, demonstrated by nasal instillation of T-1824. Re-drawn from Yoffey and Drinker (1938).

The right hand figure is for orientation, and shows only the lower end of the deep pathway, which is shown in its entirety in the figure on the left.

The entire complex may be termed the deep cervical pathway, and its main drainage area is the nose. The nasal instillation of T-1824 outlines the pathway a vivid blue, and indicates a pathway actually functioning. It constitutes a much more effective demonstration than the classical technique of interstitial injection, where the dye spreads in all directions from the site of injection, and gives no indication of the course taken by the lymph during life.

\section{The Course of Air through the Nose}

In the nose of the adult, inspired air is believed to traverse mainly the inferior meatus, and to a lesser extent the middle meatus, as well as flowing over the surfaces of the middle and inferior conchae. In fig. 4 the inspiratory current is indicated by solid arrows. The upper reaches of the nasal cavity, lined by the specialized olfactory mucosa, are above the inspiratory current and are not subject to the direct impact of inspired air. This would seem to follow from simple physical considerations, which also suggest that on a deep and powerful inspiration the rush of air against the sphenoid might cause some eddies in the roof of the nasal cavity. This is supported by the fact that one way of ensuring that air reaches the olfactory mucosa is by a powerful and deep inspiration. The other way in which inspired air may be manipulated for purposes of olfaction is to draw a small amount into the nose, and then impel it to the upper olfactory areas either by a gentle expiratory effort of brief duration, or by raising the soft palate. The first method, olfaction by deep inspiration, is the one adopted when the inspired air is known to be harmless, for example, filling one's lungs with a deep breath of country air; while the second method, sampling only small 
quantities of air, is the one adopted when dealing with air about whose qualities one is in doubt.

Because of its position outside the main inspiratory current, the olfactory mucosa is far less prone to primary infection, and in fact is usually sterile ; furthermore, it does not require the same degree of vascularity as the rest of the nasal musoca, so that it tends to be somewhat pale, with a yellowish tinge. In expiration, rather more air may reach the roof of the nose than in inspiration, the air being propelled upwards by the expiratory vis a tergo, and not drawn through the lower part of the nasal cavity by suction at the posterior nares. But it is air which is already moistened and warmed, and freed from its particulate matter. The direction of the expiratory air current is indicated in fig. 4 by broken arrows. In the newborn infant (fig. 5) the vertical depth of the nasal cavity is much less, and the posterior nares are narrower not only absolutely, but also relatively, so that presumably even inspired air has access to the superior meatus.

\section{Absorption through the Nasal Mucosa}

The passage of infective matter through the nasal mucosa is a special and peculiar instance of a much wider problem, namely that of absorption through the mucosa. At the commencement of vertebrate evolution, in fishes, the nose was part of the alimentary tract, and nose and mouth formed a single cavity. Though at a later period the development of the palate came to prevent ingested matter from coming into contact with the nasal mucosa, the latter has still retained certain powers of absorption.

As with the remainder of the alimentary canal, the first barrier to be passed by substances undergoing absorption is the mucous membrane itself, for the most part ciliated and columnar. It is perhaps to be emphasized that in the opinion of most observers the mucous membrane is a continuous layer, and does not possess the ' stomata. described by Baum and Trautmann (1925-26). Once substances are through the mucous membrane, they are in close proximity to both blood and lymph vessels. Here one would expect that simple crystalloids and water could be readily absorbed through the blood capillaries, while somewhat larger molecules could be absorbed by both blood and lymph capillaries; the larger the molecule, the greater the lymphatic absorption and the less the absorption into blood capillaries.

Up to a point, experimental work bears out this concept. If the nose is irrigated with normal saline, there is certainly very little absorption via the lymph stream (McCarrell, 1939), though curiously enough distilled water causes a marked increase in the cervical lymph flow, which at the same time shows evidence of water absorption in the form of 
even though the cervical pathway may be quite deeply stained.

Whether proteins are absorbed after nasal instillation depends upon their molecular weight. Thus egg albumen (molecular weight 34,000 ) can be readily detected in cervical lymph, serum albumin (c. 70,000) usually appears in traces, whereas serum globulin, as also horse serum, is never found in cervical lymph after nasal instillation, and presumably this is because it does not traverse the mucosal barrier (Yoffey et al., 1938). For if once it got through the mucosa it would be bound to enter the lymphatics, as is the case with any subcutaneous injection of serum.

The special property of the nasal mucosa in permitting the ready passage of T-1824 but not of horse serum has been used to demonstrate that when the dye is dissolved in horse serum the two become firmly associated. A 1 per cent. solution of T-1824 in normal saline, after nasal instillation, readily passes through the mucosa and can be identified in cervical lymph, but, if the dye is dissolved in horse serum, none appears in the lymph. However, if a 5 per cent. solution of dye in horse serum is employed, some dye does appear in the cervical lymph: presumably there is in the stronger solution an excess of dye which is not combined with the protein, and it is this uncombined dye which is free to pass through the mucosa.

\section{Viruses and Bacteria}

Ascending the scale of particle sizes, we find an essential difference between inanimate and living particles. Thus indian ink particles never penetrate the healthy mucosa, whereas viruses and bacteria may do so. This is more likely if one introduces pathogenic bacteria or viruses into a suitable animal.

It has been shown, for example (Schultz et al., 1938) that in the rabbit, which is very susceptible to pneumococcal infection, type III pneumococci readily traverse the nasal mucosa and appear in the cervical lymph. It is clear, of course, that the path taken by a highly virulent organism is determined not merely by particle size, but by complicating factors such as damage to the living mucosal and other cells. In these experiments the pneumococci were found in the lymph after it had already traversed the main cervical lymph node and was about to enter the blood stream. The experiments thus bring out the fact that in the case of virulent organisms lymph nodes do not constitute an effective barrier to the passage of bacteria.

With regard to viruses, Yoffey and Sullivan (1939) investigated the fate of vaccinia virus after nasal instillation in rabbit and monkey. From the point of view of size, virus particles are too large to pass through the mucosa. However, the virus can first establish itself in the mucosa and propagate, until presumably some invades the submucosa and then enters the lymphatics. Twelve hours after nasal instillation virus could readily be detected in cervical lymph, in which it was found at any subsequent period up to seven days after the primary infection of the mucosa. In all probability it would still have been detectable had the experiments continued over a longer period. Since the nasopharynx is so readily invaded by many viruses, the implications of these experiments are of considerable interest. It is clear that, from a nasal infection, a steady stream of virus can enter the blood and be disseminated throughout the body. In fact, it is difficult to see how, unless a virus has a specific tropism, this state of affairs could be avoided. Presumably a cold in the nose always becomes a generalized virus infection and is not limited to the nose itself.

The free and continuous passage of virus through the cervical lymph nodes is fundamentally different from the passage of bacteria, and depends upon the fact that the cytotropic virus particles become fixed to the cells of the lymph node. Most of these cells are lymphocytes which are proliferating and leaving the node to reach the blood stream. The lymphocytes thus act as virus carriers and very effectively disseminate virus throughout the body. As far as virus particles are concerned, therefore, lymph nodes not only do not afford a barrier to their spread, but on the contrary serve as a centre for the spread of infection. Furthermore, since a small number of lymphocytes is continually escaping from the blood capillaries to enter the connective tissue and the lymphatic capillaries all over the body (Yoffey and Drinker, 1939b), every lymph node in the body is in a position to become infected and serve as a further centre for virus spread. The same sequence of events presumably occurs in Jennerian vaccination, where enlargement and tenderness of the regional lymph nodes are usually easy to observe. The fact that the virus particles are carried by the lymphocytes, and are not free in the lymph, can readily be shown by centrifuging the lymph at low speeds. The virus-containing lymphocytes are thrown down, whereas the supernatant fluid does not contain virus. The lymphocytes can be repeatedly washed in saline without losing their virus.

It must be emphasized, however, that the lymphatic dissemination of viruses cannot apply to viruses with highly specific cytotropisms, for example, the neurotropic.

The Nose and the Interior of the Cranium

The roof of the nose is separated from the interior of the cranium by a thin sheet of tissue, namely the cribriform plate of the ethmoid bone, with dura-arachnoid on its cranial aspect, and nasal mucosa with submucous tissue on its nasal side. The entire tissue mass is of the order of thickness of 0.5-1.0 mm. in the adult and even less in the infant, and would seem to offer very serious possibilities for the direct spread of infection, through tissue continuity, from the nose to the cranium. The work of Rake (1937) was perhaps unnecessarily alarmist in this respect, particularly 
since, in the adult, inspired air does not have access to the superior meatus, which is usually sterile. In the newborn infant the air with its bacteria has readier access to the superior meatus. This fact, in conjunction with the thinner and softer tissue barrier, since the cribriform plate is not ossified for some little time after birth, accounts no doubt for the greater ease with which in the infant infection may spread from the nose to the interior of the cranium. Even in the adult certain types of infection are believed frequently to reach the brain by this route. Among bacteria, one may instance the meningococcus.

As far as viruses are concerned, the specifically neurotropic viruses were once thought to have in the roof of the nose a particularly ready entry to the nervous system. The free processes of the olfactory nerve cells, projecting naked and unprotected into the cavity of the nose, seemed to afford an easy contact between neurotropic viruses and nervous tissue, with rapid spread along the fibres of the olfactory nerve, through the foramina in the cribriform plate, and so via the olfactory bulbs and tracts to the brain and spinal cord. It is indeed not so very long since attempts were made to destroy the olfactory nerve endings by dropping tannic acid into the nose, and producing thereby varying degrees of permanent anosmia, in an effort to prevent the dreaded virus of poliomyelitis from reaching the nervous system. However, we now know that this heroic sacrifice of one of the special senses was in vain, since the portal of entry of the virus is elsewhere, however plausible the hypothesis of olfactory spread may once have sounded.

But if viruses or bacteria do not spread by direct propagation along the individual nerve fibres, there is theoretically another route open to them, namely the spaces around the olfactory nerve bundles, and between their constituent fibres.

The emerging bundles of the olfactory nerve are surrounded by a space containing cerebrospinal fluid and continuous with the cranial subarachnoid space. This perineural space is bounded by a thin but definite condensation of connective tissue, which accompanies the nerve as far as the submucosa, where it ceases. This is opposed to the classical idea, according to which the pia mater was closely attached to the nerve as it passed through the plate, while the dura-arachnoid also passed through the foramen to fuse just below it with the pia. In this latter way there would be a wedge-like prolongation of the subarachnoid space around the olfactory nerve (Yoffey and Drinker, 1939a) as opposed to the newer concept (Field et al., 1948).

If an animal is anaesthetized and placed on its back, a solution of trypan blue or T-1824 dropped into the nose forms a pool in direct contact with the mucous membrane immediately below the cribriform plate. The dye quickly passes through the mucosa and enters the cervical lymphatic pathway, but never reaches the interior of the cranium, even though the animal is left on its back for as long as six hours. Now, whereas these colloidal dyes do not find their way into the cranium, a crystalloid such as potassium ferrocyanide readily does so (Le Gros Clark, 1929). Yoffey and Drinker (1939a) explained these facts by assuming that the dura arachnoid sleeve had the properties of a semi-permeable membrane through which there was always a slight downward or centrifugal flow of cerebrospinal fluid into the submucous tissue of the nose. Colloids could not pass through this membrane, whereas crystalloids could, and, if their rate of diffusion was greater than that of the centrifugal flow of cerebrospinal fluid, could easily enter the cranium. On this basis it is clear that crystalloids in the cerebrospinal fluid could very readily find their way into the submucosa.

However, in view of the revised concept of Field et al. (1948), this explanation needs to be modified somewhat. The critical region is the distal part of the olfactory nerve bundle, close to the mucosa, where there is apparently free communication between the submucous tissue spaces and the perineural space, along which crystalloids or colloids (or for that matter even particles) could ascend quite freely. But they would have to make the passage against the current of cerebrospinal fluid, and apparently it is only the crystalloids which can do this, since their diffusion rate is greater than that of the fluid.

What of movement in the reverse direction, namely from the cranial subarachnoid space down to the nose? In this case the movement is with the current, not against it, and it is not surprising that not only crystalloids, but also colloidal dyes, can pass from the cranial subarachnoid space to the submucosa of the nose, and thence be carried away by the blood vessels and lymphatics. This occurs with great ease even if the pressure of the cerebrospinal fluid is kept within physiological limits, and, in fact, if dyes are used, it is the best of all methods for delineating the entire nasal lymphatic pathway.

In regard to particulate matter, it has in the past been generally accepted that within normal limits of cerebrospinal fluid pressure, particles will not pass from the cranial subarachnoid space to the nose. In the absence of information concerning particle size, these statements are difficult to assess. Recently, Field et al. (1948), found that, in the rabbit, indian ink particles (average size $0 \cdot 5 \mu$ ) could readily pass out of the cranium along the perineural spaces of the olfactory nerve bundles, and also track with great ease between their fasciculi at pressures which were carefully kept within the normal range.

The implication is that in any condition where there are bacteria or viruses in the cranial cerebrospinal fluid these may make their way to the nasal mucosa and the cervical lymphatic pathway, unless inflammation obstructs the flow of fluid in the perineural spaces. Bacteria might or might not be held back by the lymph glands of the cervical chain; but viruses, unless possessed of some very specific 
tropisms, would pass through and infect the general blood stream. It was the frequent findings of virus in the naso-pharyngeal mucosa which convinced so many of the eartier workers on poliomyelitis that the nose must be the portal of entry. In the light of our recent work, it is more likely that the virus finds its way to the nose secondarily via the cerebrospinal fluid.

\section{Practical Applicatious}

In considering absorption from the nasopharynx, the question arises whether this property of the mucosa can be put to any practical use, or has any consequences of clinical significance. The answer seems to be that while absorption definitely occurs it is not on a large scale, and therefore only substances which are potent in small amounts can have any effect. An excellent example is a substance such as mecholyl (van Dellen et al., 1937), evidence of whose absorption is provided by the rapid physiological response of the organism. The classical studies of Blumgart (1922) on pituitary extract were followed by immediate practical application of the fact of nasopharyngeal absorption. In animal experiments it is possible to isolate the nasopharynx and ensure that it is the sole site of absorption. In man, however, the use of a nasal spray, as in the work of Peters and Allison (1929) on the production of immunity to scarlet fever toxin, introduces the added complication of inhalation and swallowing, so that one cannot be certain that one is dealing exclusively with absorption through the nose.

In the case of proteins, those of larger molecular weight can be disregarded. To this class, unfortunately, belong bacterial antibodies, consisting of the globulins. Proteins of lower molecular weight, however, even though absorbed in only small amounts, may under certain conditions give rise to very definite effects. For example, if sensitization to a foreign protein has occurred, the absorption of even slight amounts may cause severe anaphylactic symptoms, either local, or general. Hay fever would appear to be a case in point.

The absorption of toxins is not perhaps strictly comparable with that of indifferent substances, since toxins may have an injurious effect on the mucosa and the endothelium of the capillaries, whereby the process of absorption will be facilitated. With this proviso, there is ample evidence to show that diphtheria toxin can be absorbed in amounts sufficient to evoke a fair degree of immunity. In this connexion one may cite the earlier work of Dserzgowsky (1910) in the horse, and of Blumenau (1911) in man. More recently, Fraser et al. (1940) have elicited an antitoxin response to concentrated diphtheria toxoid applied to the nasal mucous membrane on small pledgets of absorbent cotton wool. The pledget was placed between the anterior end of the inferior turbinate and the septum, so that apparently absorption must have been purely nasal.

As far as bactericidal substances are concerned, there is little direct evidence of their absorption. But it is clear that if a molecule as large as that of egg albumen can be absorbed without great difficulty, and because of its size pass into the cervical lymphatic pathway rather than undergo direct absorption into the blood, then some of the newer antibiotics may possibly find a use in dealing with infections of the cervical lymph glands. The determining factor (apart, that is, from the properties of the antibiotic itself) is molecule size. If the molecule is too small, absorption will occur through the blood vessels of the submucosa, and hardly any via the lymphatics. What is needed is a molecule whose size will not be too great for it to pass through the nasal mucosa, but will be too big for it to be absorbed through the submucous blood vessels, so that absorption can only occur through the cervical lymphatic pathway. The peculiar arrangement of the cervical lymph glands in man, in the form of a chain through each member of which the lymph of the pathway must pass on its way to the blood, seems to lend itself admirably to disinfection of the entire chain if only the appropriate substance could be introduced into the lymph flowing from the nose.

\section{The Cerebrospinal Fuid and the Nasal Mroosa}

The passage of ink particles from the cranial subarachnoid space, in the perineural spaces of the olfactory nerves, suggests an appreciable centripetal flow of cerebrospinal fluid. We do not as yet possess any quantitative data on this point. Weed is generally credited with stating that 95 per cent. of the cerebrospinal fluid was absorbed through the arachnoid granulations and the venous sinuses, and only 5 per cent. would then be left for all other channels of outflow, including the perineural spaces of the olfactory nerves. I have been quite unable to trace the source of the statement, or the evidence on which it is based. But whatever the quantity of fluid escaping this way, it is finally delivered close to the nasal mucosa, in the olfactory area, and it may well be that some of it passes through the mucosa to reach its surface, and is mainly responsible for keeping this region of the mucosa continually moist. There is nothing intrinsically unreasonable in this, for if molecules such as egg albumen and serum albumen can pass through the mucosa, then water and crystalloids should certainly be able to do so. The concept, if substantiated, would bring us very close to the old Greek idea of the pituita. But however attractive it may be, whether scientifically or historically, it is still hypothetical, and lacks clear experimental proof.

It is a pleasure to acknowledge my indebtedness to Dr. J. B. Brierley for the preparation of figs. 3, 4, and 5, and to Mr. J. E. Dann and Mr. J. E. Hancock for the photographs. 


\section{REFERENCES}

Baum, H., and Trautmann, A. Anat. Anz., 60, 61. Blumenau, N. R. (1911). Jb. Kinderheilk., 74, 141.

Blumgart, H. L. (1922). Arch. intern. Med., 29, 508.

Clark, W. E. Le Gros (1929). ' Report to the Committee on Vaccination on an anatomical investigation into the routes by which infections may pass from the nasal cavities into the brain.' Rep. on Pub. Health and Med. Subjects, No. 54. Ministry of Health, H.M. Stationery Office, London.

Dserzgowsky, S. K. (1910). Z. Immun. Forsch., p. 602.

Field, E. J., Brierley, J. B., and Yoffey, J. M. (1948). Proc. Anat. Soc., J. Anat. Lond. (1949.) 83, 77.

Fraser, D. T., Davey, E. L., and Halpern, K. C. (1940). Canad. J. publ. Hlth., 31, 376.
McCarrell, J. D. (1939). Amer. J. Physiol., 126, 20.

Peters, B. A., and Allison, S. F. (1929). Lancet, 1, 1035.

Rake, G. (1937). J. exp. Med., 65, 303.

Schultz, R. Z., Warren, M. F., and Drinker, C. K. (1938). J. exp. Med., 68, 251.

Van Dellen, T. R., Bruger, M., and Wright, I. S. (1937). J. Pharmacol., 59, 413.

Yoffey, J. M., and Drinker, C. K. (1938). J. exp. Med., 68,629 .

(1939a). J. Anat., Lond., 74, 45.

(1939b). Anat. Rec., 73, 417.

and Sullivan, E. R. (1939). J. exp. Med., 69, 133. , and Drinker, C. K. (1938). J. exp. Med., 68, 941 . 